\title{
On a product operator from weighted Bergman-Nevanlinna spaces to weighted Zygmund spaces
}

Zhi-jie Jiang ${ }^{*}$

"Correspondence: matjzj@126.com Institute of Nonlinear Science and Engineering Computing, Sichuan

University of Science and Engineering, Zigong, Sichuan 643000 , P.R. China

\begin{abstract}
Let $\mathbb{D}=\{z \in \mathbb{C}:|z|<1\}$ be the open unit disk, $\varphi$ an analytic self-map of $\mathbb{D}$ and $\psi$ an analytic function in $\mathbb{D}$. Let $\mathcal{D}$ be the differentiation operator and $W_{\varphi, \psi}$ the weighted composition operator. The boundedness and compactness of the product operator $\mathcal{D} W_{\varphi, \psi}$ from weighted Bergman-Nevanlinna spaces to weighted Zygmund spaces on $\mathbb{D}$ are characterized

MSC: Primary 47B38; secondary 47B33; 47B37

Keywords: weighted Bergman-Nevanlinna space; product operator; weighted Zygmund space; little weighted Zygmund space
\end{abstract}

\section{Introduction}

Let $\mathbb{C}$ be the complex plane, $\mathbb{D}=\{z \in \mathbb{C}:|z|<1\}$ the open unit disk, $H(\mathbb{D})$ the space of all analytic functions on $\mathbb{D}$ and $d A(z)=\frac{1}{\pi} d x d y$ the normalized Lebesgue measure on $\mathbb{D}$. For $\alpha>-1$, let $d A_{\alpha}(z)=(\alpha+1)\left(1-|z|^{2}\right)^{\alpha} d A(z)$ be the weighted Lebesgue measure on $\mathbb{D}$. The weighted Bergman-Nevanlinna space $\mathcal{A}_{\log }^{\alpha}$ consists of all $f \in H(\mathbb{D})$ such that

$$
\|f\|_{\mathcal{A}_{\log }^{\alpha}}=\int_{\mathbb{D}} \log (1+|f(z)|) d A_{\alpha}(z)<\infty .
$$

It is a Fréchet space with the translation invariant metric

$$
d(f, g)=\|f-g\|_{\mathcal{A}_{\log }^{\alpha}} .
$$

For some details of this space, see, e.g., [1-3] and [4].

For $\beta>0$, the weighted-type $\mathcal{A}_{\beta}$ consists of all $f \in H(\mathbb{D})$ such that

$$
\sup _{z \in \mathbb{D}}\left(1-|z|^{2}\right)^{\beta}|f(z)|<\infty .
$$

This space is a non-separable Banach space with the norm defined by

$$
\|f\|_{\mathcal{A}_{\beta}}=\sup _{z \in \mathbb{D}}\left(1-|z|^{2}\right)^{\beta}|f(z)| .
$$

O2014 Jiang; licensee Springer. This is an Open Access article distributed under the terms of the Creative Commons Attribution License (http://creativecommons.org/licenses/by/2.0), which permits unrestricted use, distribution, and reproduction in any medium, provided the original work is properly cited. 
The closure of the set of polynomials in $\mathcal{A}_{\beta}$ is denoted by $\mathcal{A}_{\beta, 0}$, which is a separable Banach space and consists exactly of those functions $f$ in $\mathcal{A}_{\beta}$ satisfying the condition

$$
\lim _{|z| \rightarrow 1^{-}}\left(1-|z|^{2}\right)^{\beta}|f(z)|=0
$$

For $\beta>0$, the weighted Bloch space is defined by

$$
\mathcal{B}_{\beta}=\left\{f \in H(\mathbb{D}): \sup _{z \in \mathbb{D}}\left(1-|z|^{2}\right)^{\beta}\left|f^{\prime}(z)\right|<\infty\right\},
$$

and it is well known (see, e.g., [5]) that, if $\beta>1$, then $f \in \mathcal{B}_{\beta}$ if and only if $f \in \mathcal{A}_{\beta-1}$. Under the norm

$$
\|f\|_{\mathcal{B}_{\beta}}=|f(0)|+\sup _{z \in \mathbb{D}}\left(1-|z|^{2}\right)^{\beta}\left|f^{\prime}(z)\right|
$$

it is a Banach space. The closure of the set of polynomials in $\mathcal{B}_{\beta}$ is called the little weighted Bloch space and denoted by $\mathcal{B}_{\beta, 0}$. For a good source for such spaces, we refer to [5].

For $\beta>0$, the weighted Zygmund space $\mathcal{Z}_{\beta}$ consists of all $f \in H(\mathbb{D})$ such that

$$
\sup _{z \in \mathbb{D}}\left(1-|z|^{2}\right)^{\beta}\left|f^{\prime \prime}(z)\right|<\infty
$$

It is a Banach space with the norm

$$
\|f\|_{\mathcal{Z}_{\beta}}=|f(0)|+\left|f^{\prime}(0)\right|+\sup _{z \in \mathbb{D}}\left(1-|z|^{2}\right)^{\beta}\left|f^{\prime \prime}(z)\right| .
$$

The little weighted Zygmund space $\mathcal{Z}_{\beta, 0}$ consists those functions $f$ in $\mathcal{Z}_{\beta}$ satisfying

$$
\lim _{|z| \rightarrow 1^{-}}\left(1-|z|^{2}\right)^{\beta}\left|f^{\prime \prime}(z)\right|=0
$$

and it is a closed subspace of the weighted Zygmund space.

Recently, many authors have studied the properties of some concrete operators between various spaces of analytic functions in the unit disk, the upper half plane, the unit ball and the unit polydisk. For some operators on weighted-type spaces, weighted Bloch spaces, and weighted Zygmund spaces on these domains, see, e.g., $[4,6-38]$ and the references therein.

Let $\varphi$ be an analytic self-map of $\mathbb{D}$ and let $\psi$ be an analytic function in $\mathbb{D}$. It is well known that the weighted composition operator $W_{\varphi, \psi}$ on $H(\mathbb{D})$ is defined by

$$
W_{\varphi, \psi} f(z)=\psi(z) \cdot f(\varphi(z)), \quad z \in \mathbb{D} .
$$

If $\psi \equiv 1, W_{\varphi, \psi}:=C_{\varphi}$ is called the composition operator. If $\varphi(z)=z, W_{\varphi, \psi}:=M_{\psi}$ it is called the multiplication operator. It is of interest to provide function theoretic characterizations when $\varphi$ and $\psi$ induce a bounded or compact weighted composition operator. Sharma and Abbas have studied the boundedness and compactness of weighted composition operators from weighted Bergman-Nevanlinna spaces to Bloch spaces in [39]. Kumar and Sharma 
have characterized the boundedness and compactness of weighted composition operators from weighted Bergman-Nevanlinna spaces to Zygmund spaces in [40].

Now we list several operators, which will be considered in this paper. Let $\mathcal{D}$ be the differentiation operator on $H(\mathbb{D})$ defined by

$$
\mathcal{D} f(z)=f^{\prime}(z), \quad z \in \mathbb{D} \text {. }
$$

Operator $\mathcal{D} C_{\varphi}$ has been studied, for example, in $[10,15,19,20,31,35,41-45]$. Here we want to mention that Sharma has studied the following two operators from BergmanNevanlinna spaces to Bloch-type spaces in [46]. They are defined as follows:

$$
\mathcal{D} M_{\psi} C_{\varphi} f(z)=\psi^{\prime}(z) f(\varphi(z))+\psi(z) \varphi^{\prime}(z) f^{\prime}(\varphi(z))
$$

and

$$
\mathcal{D} C_{\varphi} M_{\psi} f(z)=\varphi^{\prime}(z) \psi^{\prime}(\varphi(z)) f(\varphi(z))+\psi(\varphi(z)) \varphi^{\prime}(z) f^{\prime}(\varphi(z)),
$$

for $z \in \mathbb{D}$ and $f \in H(\mathbb{D})$. These operators on weighted Bergman spaces have been also studied by Stević et al. in [47] and [48]. If we consider the product operator $\mathcal{D} W_{\varphi, \psi}$, it is clear that $\mathcal{D} M_{\psi} C_{\varphi}=\mathcal{D} W_{\varphi, \psi}$ and $\mathcal{D} C_{\varphi} M_{\psi}=\mathcal{D} W_{\varphi, \psi \circ \varphi}$. Quite recently, the operator $\mathcal{D} W_{\varphi, \psi}$ from weighted Bergman spaces to weighted Zygmund spaces has been considered in [49]. This paper is devoted to characterizing the boundedness and compactness of the operator $\mathcal{D} W_{\varphi, \psi}$ from weighted Bergman-Nevanlinna spaces to weighted Zygmund spaces. This paper can be regarded as an continuousness of our work.

Since the weighted Bergman-Nevanlinna space is a Fréchet space and not a Banach space, it is necessary to introduce several definitions needed in this paper. Let $X$ and $Y$ be topological vector spaces whose topologies are given by translation invariant metrics $d_{X}$ and $d_{Y}$, respectively, and let $L: X \rightarrow Y$ be a linear operator. It is said that $L$ is metrically bounded if there exists a positive constant $K$ such that

$$
d_{Y}(L f, 0) \leq K d_{X}(f, 0)
$$

for all $f \in X$. When $X$ and $Y$ are Banach spaces, the metrical boundedness coincides with the usual definition of bounded operators between Banach spaces. Recall that $L: X \rightarrow Y$ is metrically compact if it maps bounded sets into relatively compact sets. When $X$ and $Y$ are Banach spaces, the metrical compactness coincides with the usual definition of compact operators between Banach spaces. When $X=\mathcal{A}_{\log }^{\alpha}$ and $Y$ is a Banach space, we define

$$
\|L\|_{\mathcal{A}_{\log }^{\alpha} \rightarrow Y}=\sup _{\|f\|_{\mathcal{A}_{\log }^{\alpha}}^{\alpha} \leq 1}\|L f\|_{Y}
$$

and we often write $\|L\|_{\mathcal{A}_{\log }^{\alpha} \rightarrow Y}$ by $\|L\|$.

Throughout this paper, an operator is bounded (respectively, compact), if it is metrically bounded (respectively, metrically compact). Constants are denoted by $C$, they are positive and may differ from one occurrence to the next. The notation $a \asymp b$ means that there exists a positive constant $C$ such that $a / C \leq b \leq C a$. 


\section{The operator $\mathcal{D} W_{\varphi, \psi}: \mathcal{A}_{\text {log }}^{\alpha} \rightarrow \mathcal{Z}_{\beta}\left(\mathcal{Z}_{\beta, 0}\right)$}

Our first lemma characterizes the compactness in terms of sequential convergence. Since the proof is standard, it is omitted (see Proposition 3.11 in [50]).

Lemma 2.1 Let $\alpha>-1, \beta>0$ and $\mathcal{Y} \in\left\{\mathcal{Z}_{\beta}, \mathcal{Z}_{\beta, 0}\right\}$. Then the bounded operator $\mathcal{D} W_{\varphi, \psi}$ : $\mathcal{A}_{\log }^{\alpha} \rightarrow \mathcal{Y}$ is compact if and only if for every bounded sequence $\left(f_{n}\right)_{n \in \mathbb{N}}$ in $\mathcal{A}_{\log }^{\alpha}$ such that $f_{n} \rightarrow 0$ uniformly on every compact subset of $\mathbb{D}$ as $n \rightarrow \infty$, it follows that

$$
\lim _{n \rightarrow \infty}\left\|\mathcal{D} W_{\varphi, \psi} f_{n}\right\|_{\mathcal{Y}}=0
$$

The next result can be found, for example, in [4].

Lemma 2.2 Let $\alpha>-1$ and $n \in \mathbb{N}_{0}=\mathbb{N} \cup\{0\}$. Then, for all $f \in \mathcal{A}_{\log }^{\alpha}$ and $z \in \mathbb{D}$, there exists a positive constant $C$ independent of $f$ such that

$$
\left(1-|z|^{2}\right)^{n}\left|f^{(n)}(z)\right| \leq \exp \frac{C\|f\|_{\mathcal{A}_{\log }^{\alpha}}}{\left(1-|z|^{2}\right)^{\alpha+2}} .
$$

Now we consider the boundedness of the operator $\mathcal{D} W_{\varphi, \psi}: \mathcal{A}_{\log }^{\alpha} \rightarrow \mathcal{Z}_{\beta}$.

Theorem 2.3 Let $\alpha>-1, \beta>0, \varphi$ an analytic self-map of $\mathbb{D}$ and $\psi \in H(\mathbb{D})$. Then, for all $c>0$, the following statements are equivalent:

(i) The operator $\mathcal{D} W_{\varphi, \psi}: \mathcal{A}_{\log }^{\alpha} \rightarrow \mathcal{Z}_{\beta}$ is bounded.

(ii) The operator $\mathcal{D} W_{\varphi, \psi}: \mathcal{A}_{\log }^{\alpha} \rightarrow \mathcal{Z}_{\beta}$ is compact.

(iii) $\psi^{\prime} \in \mathcal{Z}_{\beta}$,

$$
\begin{aligned}
& M_{0}:=\sup _{z \in \mathbb{D}}\left(1-|z|^{2}\right)^{\beta}\left|\psi(z) \varphi^{\prime \prime \prime}(z)+3 \psi^{\prime \prime}(z) \varphi^{\prime}(z)+3 \psi^{\prime}(z) \varphi^{\prime \prime}(z)\right|<\infty, \\
& M_{1}:=\sup _{z \in \mathbb{D}}\left(1-|z|^{2}\right)^{\beta}|\psi(z)|\left|\varphi^{\prime}(z)\right|^{3}<\infty, \\
& M_{2}:=\sup _{z \in \mathbb{D}}\left(1-|z|^{2}\right)^{\beta}\left|\psi^{\prime}(z) \varphi^{\prime}(z)^{2}+\psi(z) \varphi^{\prime}(z) \varphi^{\prime \prime}(z)\right|<\infty, \\
& \lim _{\varphi(z) \rightarrow \partial \mathbb{D}}\left(1-|z|^{2}\right)^{\beta}\left|\psi^{\prime \prime \prime}(z)\right| \exp \frac{c}{\left(1-|\varphi(z)|^{2}\right)^{\alpha+2}}=0, \\
& \lim _{\varphi(z) \rightarrow \partial \mathbb{D}} \frac{\left(1-|z|^{2}\right)^{\beta}}{\left(1-|\varphi(z)|^{2}\right)^{3}}|\psi(z)|\left|\varphi^{\prime}(z)\right|^{3} \exp \frac{c}{\left(1-|\varphi(z)|^{2}\right)^{\alpha+2}}=0, \\
& \lim _{\varphi(z) \rightarrow \partial \mathbb{D}} \frac{\left(1-|z|^{2}\right)^{\beta}}{\left(1-|\varphi(z)|^{2}\right)^{2}}\left|\psi^{\prime}(z) \varphi^{\prime}(z)^{2}+\psi(z) \varphi^{\prime}(z) \varphi^{\prime \prime}(z)\right| \exp \frac{c}{\left(1-|\varphi(z)|^{2}\right)^{\alpha+2}}=0,
\end{aligned}
$$

and

$$
\lim _{\varphi(z) \rightarrow \partial \mathbb{D}} \frac{\left(1-|z|^{2}\right)^{\beta}}{1-|\varphi(z)|^{2}}\left|\psi(z) \varphi^{\prime \prime \prime}(z)+3 \psi^{\prime \prime}(z) \varphi^{\prime}(z)+3 \psi^{\prime}(z) \varphi^{\prime \prime}(z)\right| \exp \frac{c}{\left(1-|\varphi(z)|^{2}\right)^{\alpha+2}}=0 .
$$

Proof (i) $\Rightarrow$ (iii). Suppose that (i) holds. Take the functions $f(z)=z$ and $f(z) \equiv 1$, respectively. Since the operator $\mathcal{D} W_{\varphi, \psi}: \mathcal{A}_{\log }^{\alpha} \rightarrow \mathcal{Z}_{\beta}$ is bounded, we have

$$
\begin{aligned}
& \sup _{z \in \mathbb{D}}\left(1-|z|^{2}\right)^{\beta}\left|\psi^{\prime \prime \prime}(z) \varphi(z)+3 \psi^{\prime \prime}(z) \varphi^{\prime}(z)+3 \psi^{\prime}(z) \varphi^{\prime \prime}(z)+\psi(z) \varphi^{\prime \prime \prime}(z)\right| \\
& \quad \leq\left\|\mathcal{D} W_{\varphi, \psi} z\right\|_{\mathcal{Z}_{\beta}} \leq C\left\|\mathcal{D} W_{\varphi, \psi}\right\|,
\end{aligned}
$$


and

$$
\sup _{z \in \mathbb{D}}\left(1-|z|^{2}\right)^{\beta}\left|\psi^{\prime \prime \prime}(z)\right| \leq\left\|\mathcal{D} W_{\varphi, \psi} 1\right\|_{\mathcal{Z}_{\beta}} \leq C\left\|\mathcal{D} W_{\varphi, \psi}\right\|
$$

Inequality (2) shows $\psi^{\prime} \in \mathcal{Z}_{\beta}$. From (1), (2), and the boundedness of $\varphi$, it follows that

$$
M_{0}=\sup _{z \in \mathbb{D}}\left(1-|z|^{2}\right)^{\beta}\left|\psi(z) \varphi^{\prime \prime \prime}(z)+3 \psi^{\prime \prime}(z) \varphi^{\prime}(z)+3 \psi^{\prime}(z) \varphi^{\prime \prime}(z)\right| \leq C\left\|\mathcal{D} W_{\varphi, \psi}\right\| .
$$

Taking the functions $f(z)=z^{2}$ and $f(z)=z^{3}$, respectively, we have

$$
\begin{aligned}
\sup _{z \in \mathbb{D}}(1 & \left.-|z|^{2}\right)^{\beta} \mid \psi(z)^{\prime \prime \prime}(z) \varphi(z)^{2}+6 \psi^{\prime \prime}(z) \varphi^{\prime}(z) \varphi(z)+6 \psi^{\prime}(z) \varphi^{\prime \prime}(z) \varphi(z) \\
& +6 \psi^{\prime}(z) \varphi^{\prime}(z)^{2}+6 \psi(z) \varphi^{\prime}(z) \varphi^{\prime \prime}(z)+2 \psi(z) \varphi^{\prime \prime \prime}(z) \varphi(z) \mid \\
\leq & \left\|\mathcal{D} W_{\varphi, \psi} z^{2}\right\|_{\mathcal{Z}_{\beta}} \leq C\left\|\mathcal{D} W_{\varphi, \psi}\right\|,
\end{aligned}
$$

and

$$
\begin{aligned}
\sup _{z \in \mathbb{D}}(1 & \left.-|z|^{2}\right)^{\beta} \mid \psi^{\prime \prime \prime}(z) \varphi(z)^{3}+9 \psi^{\prime \prime}(z) \varphi^{\prime}(z) \varphi(z)^{2}+18 \psi^{\prime}(z) \varphi(z) \varphi^{\prime}(z)^{2} \\
& +6 \psi(z) \varphi^{\prime}(z)^{3}+18 \psi(z) \varphi(z) \varphi^{\prime}(z) \varphi^{\prime \prime}(z)+9 \psi^{\prime}(z) \varphi^{\prime \prime}(z) \varphi(z)^{2}+3 \psi(z) \varphi(z)^{2} \varphi^{\prime \prime \prime}(z) \mid \\
\leq & \left\|\mathcal{D} W_{\varphi, \psi} z^{3}\right\|_{\mathcal{Z}_{\beta}} \leq C\left\|\mathcal{D} W_{\varphi, \psi}\right\| .
\end{aligned}
$$

By (2) and the boundedness of $\varphi$, we have

$$
\sup _{z \in \mathbb{D}}\left(1-|z|^{2}\right)^{\beta}\left|\psi^{\prime \prime \prime}(z)\right||\varphi(z)|^{2} \leq C\left\|\mathcal{D} W_{\varphi, \psi}\right\|
$$

and

$$
\sup _{z \in \mathbb{D}}\left(1-|z|^{2}\right)^{\beta}\left|\psi^{\prime \prime \prime}(z)\right||\varphi(z)|^{3} \leq C\left\|\mathcal{D} W_{\varphi, \psi}\right\| .
$$

From (3), (4), (6), and the boundedness of $\varphi$, it follows that

$$
M_{2}=\sup _{z \in \mathbb{D}}\left(1-|z|^{2}\right)^{\beta}\left|\psi^{\prime}(z) \varphi^{\prime}(z)^{2}+\psi(z) \varphi^{\prime}(z) \varphi^{\prime \prime}(z)\right| \leq C\left\|\mathcal{D} W_{\varphi, \psi}\right\| .
$$

Inequalities (3), (5), (7), (8), and the boundedness of $\varphi$ give

$$
M_{1}=\sup _{z \in \mathbb{D}}\left(1-|z|^{2}\right)\left|\psi(z)\left\|\left.\varphi^{\prime}(z)\right|^{3} \leq C\right\| \mathcal{D} W_{\varphi, \psi} \|\right.
$$

For $w \in \mathbb{D}$, we choose the functions

$$
\begin{aligned}
f_{1}(z)= & \frac{\alpha+4}{3 \alpha+6} \frac{\left(1-|\varphi(w)|^{2}\right)^{\alpha+2}}{(1-\overline{\varphi(w)} z)^{2(\alpha+2)}}-\frac{2 \alpha+8}{\alpha+3} \frac{\left(1-|\varphi(w)|^{2}\right)^{\alpha+4}}{(1-\overline{\varphi(w)} z)^{2(\alpha+2)+2}} \\
& +\frac{16 \alpha+64}{6 \alpha+21} \frac{\left(1-|\varphi(w)|^{2}\right)^{\alpha+5}}{(1-\overline{\varphi(w)} z)^{2(\alpha+2)+3}}-\frac{\left(1-|\varphi(w)|^{2}\right)^{\alpha+6}}{(1-\overline{\varphi(w)} z)^{2(\alpha+2)+4}}
\end{aligned}
$$


and

$$
g_{1}(z)=\frac{2 \alpha+7}{4 \alpha+8} \frac{\left(1-|\varphi(w)|^{2}\right)^{\alpha+2}}{(1-\overline{\varphi(w)} z)^{2(\alpha+2)}}-\frac{6 \alpha+21}{4 \alpha+12} \frac{\left(1-|\varphi(w)|^{2}\right)^{\alpha+4}}{(1-\overline{\varphi(w)} z)^{2(\alpha+2)+2}}+\frac{\left(1-|\varphi(w)|^{2}\right)^{\alpha+5}}{(1-\overline{\varphi(w)} z)^{2(\alpha+2)+3}}
$$

By a direct calculation, it follows that

$$
f_{1}^{\prime}(\varphi(w))=f_{1}^{\prime \prime}(\varphi(w))=f_{1}^{\prime \prime \prime}(\varphi(w))=0
$$

and

$$
g_{1}^{\prime}(\varphi(w))=g_{1}^{\prime \prime}(\varphi(w))=0
$$

Using $f_{1}$ and $g_{1}$, we define the function $f(z)=f_{1}(z) \exp c g_{1}(z)$. Applying (10) and (11) to $f^{\prime}$, $f^{\prime \prime}$ and $f^{\prime \prime \prime}$, we find

$$
f^{\prime}(\varphi(w))=f^{\prime \prime}(\varphi(w))=f^{\prime \prime \prime}(\varphi(w))=0
$$

It is obvious that

$$
f(\varphi(w))=\frac{C}{\left(1-|\varphi(w)|^{2}\right)^{\alpha+2}} \exp \frac{c}{\left(1-|\varphi(w)|^{2}\right)^{\alpha+2}},
$$

where

$$
C=\frac{\alpha+4}{3 \alpha+6}-\frac{2 \alpha+8}{\alpha+3}+\frac{16 \alpha+64}{6 \alpha+21}-1
$$

From the proof in [46], we see that $f \in \mathcal{A}_{\log }^{\alpha}$ and $\|f\|_{\mathcal{A}_{\log }^{\alpha}} \leq C$. Since the operator $\mathcal{D} W_{\varphi, \psi}$ : $\mathcal{A}_{\log }^{\alpha} \rightarrow \mathcal{Z}_{\beta}$ is bounded, it follows that

$$
\left\|\mathcal{D} W_{\varphi, \psi} f\right\|_{\mathcal{Z}_{\beta}} \leq C\left\|\mathcal{D} W_{\varphi, \psi}\right\|
$$

which means that, for all $z \in \mathbb{D}$,

$$
\left(1-|z|^{2}\right)^{\beta}\left|\left(\mathcal{D} W_{\varphi, \psi} f\right)^{\prime \prime}(z)\right| \leq C .
$$

Replacing $z$ by $w$ in (12), we obtain

$$
\frac{\left(1-|w|^{2}\right)^{\beta}}{\left(1-|\varphi(w)|^{2}\right)^{\alpha+2}}\left|\psi^{\prime \prime \prime}(w)\right| \exp \frac{c}{\left(1-|\varphi(w)|^{2}\right)^{\alpha+2}} \leq C
$$

and then

$$
\left(1-|w|^{2}\right)^{\beta}\left|\psi^{\prime \prime \prime}(w)\right| \exp \frac{c}{\left(1-|\varphi(w)|^{2}\right)^{\alpha+2}} \leq C\left(1-|\varphi(w)|^{2}\right)^{\alpha+2}
$$

Taking the limit as $\varphi(w) \rightarrow \partial \mathbb{D}$ in (13), it gives

$$
\lim _{\varphi(w) \rightarrow \partial \mathbb{D}}\left(1-|w|^{2}\right)^{\beta}\left|\psi^{\prime \prime \prime}(w)\right| \exp \frac{c}{\left(1-|\varphi(w)|^{2}\right)^{\alpha+2}}=0 .
$$


For $w \in \mathbb{D}$, we choose the functions

$$
\begin{aligned}
f_{2}(z)= & \frac{1}{3} \frac{\left(1-|\varphi(w)|^{2}\right)^{\alpha+2}}{(1-\overline{\varphi(w)} z)^{2(\alpha+2)}}-2 \frac{\left(1-|\varphi(w)|^{2}\right)^{\alpha+4}}{(1-\overline{\varphi(w)} z)^{2(\alpha+2)+2}} \\
& +\frac{8}{3} \frac{\left(1-|\varphi(w)|^{2}\right)^{\alpha+5}}{(1-\overline{\varphi(w)} z)^{2(\alpha+2)+3}}-\frac{\left(1-|\varphi(w)|^{2}\right)^{\alpha+6}}{(1-\overline{\varphi(w)} z)^{2(\alpha+2)+4}}
\end{aligned}
$$

and

$$
g_{2}(z)=\frac{\left(1-|\varphi(w)|^{2}\right)^{\alpha+2}}{(1-\overline{\varphi(w)} z)^{2(\alpha+2)}}
$$

From a calculation, we obtain

$$
f_{2}(\varphi(w))=f_{2}^{\prime}(\varphi(w))=f_{2}^{\prime \prime}(\varphi(w))=0
$$

Define the function $g(z)=f_{2}(z) \exp c g_{2}(z)$. Then by (14),

$$
g(\varphi(w))=g^{\prime}(\varphi(w))=g^{\prime \prime}(\varphi(w))=0
$$

and by a direct calculation,

$$
g^{\prime \prime \prime}(\varphi(w))=C \frac{\overline{\varphi(w)}^{3}}{\left(1-|\varphi(w)|^{2}\right)^{\alpha+5}} \exp \frac{c}{\left(1-|\varphi(w)|^{2}\right)^{\alpha+2}}
$$

where $C=-30(\alpha+2)^{2}-8$. Since $\mathcal{D} W_{\varphi, \psi}: \mathcal{A}_{\log }^{\alpha} \rightarrow \mathcal{Z}_{\beta}$ is bounded, we have

$$
\left\|\mathcal{D} W_{\varphi, \psi} g\right\|_{\mathcal{Z}_{\beta}} \leq C\left\|\mathcal{D} W_{\varphi, \psi}\right\|
$$

and so

$$
\left(1-|z|^{2}\right)^{\beta}\left|\left(\mathcal{D} W_{\varphi, \psi} g\right)^{\prime \prime}(z)\right| \leq C\left\|\mathcal{D} W_{\varphi, \psi}\right\|
$$

for all $z \in \mathbb{D}$. Letting $z=w$ in (15) yields

$$
\frac{\left(1-|w|^{2}\right)^{\beta}\left|\varphi^{\prime}(w)\right|^{3}|\varphi(w)|^{3}|\psi(w)|}{\left(1-|\varphi(w)|^{2}\right)^{\alpha+5}} \exp \frac{c}{\left(1-|\varphi(w)|^{2}\right)^{\alpha+2}} \leq C\left\|W_{\varphi, \psi}\right\| .
$$

Thus

$$
\frac{\left(1-|w|^{2}\right)^{\beta}}{\left(1-|\varphi(w)|^{2}\right)^{3}}\left|\varphi^{\prime}(w)\right|^{3}|\psi(w)| \exp \frac{c}{\left(1-|\varphi(w)|^{2}\right)^{\alpha+2}} \leq \frac{C\left(1-|\varphi(w)|^{2}\right)^{\alpha+2}}{|\varphi(w)|^{3}}
$$

Taking the limit as $\varphi(w) \rightarrow \partial \mathbb{D}$ in (16), we have

$$
\lim _{\varphi(w) \rightarrow \partial \mathbb{D}} \frac{\left(1-|w|^{2}\right)^{\beta}}{\left(1-|\varphi(w)|^{2}\right)^{3}}\left|\varphi^{\prime}(w)\right|^{3}|\psi(w)| \exp \frac{c}{\left(1-|\varphi(w)|^{2}\right)^{\alpha+2}}=0 .
$$


For $w \in \mathbb{D}$, we choose the function

$$
\begin{aligned}
f_{3}(z)= & c_{1} \frac{\left(1-|\varphi(w)|^{2}\right)^{\alpha+2}}{(1-\overline{\varphi(w)} z)^{2(\alpha+2)}}+c_{2} \frac{\left(1-|\varphi(w)|^{2}\right)^{\alpha+4}}{(1-\overline{\varphi(w)} z)^{2(\alpha+2)+2}} \\
& +c_{3} \frac{\left(1-|\varphi(w)|^{2}\right)^{\alpha+5}}{(1-\overline{\varphi(w)} z)^{2(\alpha+2)+3}}-\frac{\left(1-|\varphi(w)|^{2}\right)^{\alpha+6}}{(1-\overline{\varphi(w)} z)^{2(\alpha+2)+4}}
\end{aligned}
$$

where

$$
c_{2}=-\frac{48 \alpha^{3}+460 \alpha^{2}+1398 \alpha+1340}{24 \alpha^{3}+214 \alpha^{2}+655 \alpha+682}, \quad c_{3}=\frac{16 \alpha^{2}+104 \alpha+164}{6 \alpha^{2}+37 \alpha+62},
$$

and

$$
c_{1}=1-c_{2}-c_{3} \text {. }
$$

We also choose the function

$$
g_{3}(z)=\frac{2 \alpha+7}{4 \alpha+8} \frac{\left(1-|\varphi(w)|^{2}\right)^{\alpha+2}}{(1-\overline{\varphi(w)} z)^{2(\alpha+2)}}-\frac{6 \alpha+21}{4 \alpha+12} \frac{\left(1-|\varphi(w)|^{2}\right)^{\alpha+4}}{(1-\overline{\varphi(w)} z)^{2(\alpha+2)+2}}+\frac{\left(1-|\varphi(w)|^{2}\right)^{\alpha+5}}{(1-\overline{\varphi(w)} z)^{2(\alpha+2)+3}}
$$

For the functions $f_{3}$ and $g_{3}$, we have

$$
f_{3}(\varphi(w))=f_{3}^{\prime \prime}(\varphi(w))=f_{3}^{\prime \prime \prime}(\varphi(w))=0
$$

and

$$
g_{3}^{\prime}(\varphi(w))=g_{3}^{\prime \prime}(\varphi(w))=0
$$

Consequently, (17) and (18) make the function $h(z)=f_{3}(z) \exp c g_{3}(z)$ to satisfy

$$
h(\varphi(w))=h^{\prime \prime}(\varphi(w))=h^{\prime \prime \prime}(\varphi(w))=0
$$

and

$$
h^{\prime}(\varphi(w))=C \frac{\overline{\varphi(w)}}{\left(1-|\varphi(w)|^{2}\right)^{\alpha+3}} \exp \frac{c}{\left(1-|\varphi(w)|^{2}\right)^{\alpha+2}}
$$

where

$$
C=2 c_{2}+3 c_{3}-4
$$

By the boundedness of the operator $\mathcal{D} W_{\varphi, \psi}: \mathcal{A}_{\log }^{\alpha} \rightarrow \mathcal{Z}_{\beta}$, we find

$$
\begin{aligned}
& \frac{|\varphi(w)|\left(1-|w|^{2}\right)^{\beta}}{\left(1-|\varphi(w)|^{2}\right)^{\alpha+3}}\left|3 \psi^{\prime \prime}(w) \varphi^{\prime}(w)+3 \psi^{\prime}(w) \varphi^{\prime \prime}(w)+\psi(w) \varphi^{\prime \prime \prime}(w)\right| \\
& \quad \times \exp \frac{c}{\left(1-|\varphi(w)|^{2}\right)^{\alpha+2}} \leq C .
\end{aligned}
$$


Thus

$$
\begin{aligned}
& \lim _{\varphi(w) \rightarrow \partial \mathbb{D}} \frac{\left(1-|w|^{2}\right)^{\beta}}{1-|\varphi(w)|^{2}}\left|3 \psi^{\prime \prime}(w) \varphi^{\prime}(w)+3 \psi^{\prime}(w) \varphi^{\prime \prime}(w)+\psi(w) \varphi^{\prime \prime \prime}(w)\right| \\
& \quad \times \exp \frac{c}{\left(1-|\varphi(w)|^{2}\right)^{\alpha+2}}=0 .
\end{aligned}
$$

For $w \in \mathbb{D}$, we choose the functions

$$
\begin{aligned}
f_{4}(z)= & \frac{3 \alpha+8}{3 \alpha+10} \frac{\left(1-|\varphi(w)|^{2}\right)^{\alpha+2}}{(1-\overline{\varphi(w)} z)^{2(\alpha+2)}}-\frac{6 \alpha+22}{3 \alpha+10} \frac{\left(1-|\varphi(w)|^{2}\right)^{\alpha+4}}{(1-\overline{\varphi(w)} z)^{2(\alpha+2)+2}} \\
& +\frac{6 \alpha+24}{3 \alpha+10} \frac{\left(1-|\varphi(w)|^{2}\right)^{\alpha+5}}{(1-\overline{\varphi(w)} z)^{2(\alpha+2)+3}}-\frac{\left(1-|\varphi(w)|^{2}\right)^{\alpha+6}}{(1-\overline{\varphi(w)} z)^{2(\alpha+2)+4}}
\end{aligned}
$$

and

$$
g_{4}(z)=\frac{\alpha+3}{\alpha+2} \frac{\left(1-|\varphi(w)|^{2}\right)^{\alpha+2}}{(1-\overline{\varphi(w)} z)^{2(\alpha+2)}}-\frac{\left(1-|\varphi(w)|^{2}\right)^{\alpha+4}}{(1-\overline{\varphi(w)} z)^{2(\alpha+2)+2}}
$$

Then

$$
f_{4}(\varphi(w))=f_{4}^{\prime}(\varphi(w))=f_{4}^{\prime \prime \prime}(\varphi(w))=0
$$

and $g_{4}^{\prime}(\varphi(w))=0$. From this and (19), for the function $u(z)=f_{4}(z) \exp c g_{4}(z)$ we have

$$
u(\varphi(w))=u^{\prime}(\varphi(w))=u^{\prime \prime \prime}(\varphi(w))=0
$$

and

$$
u^{\prime \prime}(\varphi(w))=C \frac{\overline{\varphi(w)}^{2}}{\left(1-|\varphi(w)|^{2}\right)^{\alpha+4}} \exp \frac{c}{\left(1-|\varphi(w)|^{2}\right)^{\alpha+2}},
$$

where

$$
C=-\frac{24 \alpha+120 \alpha+141}{3 \alpha+10}
$$

By the boundedness of $\mathcal{D} W_{\varphi, \psi}: \mathcal{A}_{\log }^{\alpha} \rightarrow \mathcal{Z}_{\beta}$,

$$
\left\|\mathcal{D} W_{\varphi, \psi} u_{\varphi(w)}\right\|_{\mathcal{Z}_{\beta}} \leq C\left\|\mathcal{D} W_{\varphi, \psi}\right\|
$$

and from which we get

$$
\frac{|\varphi(w)|^{2}\left(1-|w|^{2}\right)^{\beta}}{\left(1-|\varphi(w)|^{2}\right)^{\alpha+4}}\left|\psi^{\prime}(w) \varphi^{\prime}(w)^{2}+\psi(w) \varphi^{\prime}(w) \varphi^{\prime \prime}(w)\right| \exp \frac{c}{\left(1-|\varphi(w)|^{2}\right)^{\alpha+2}} \leq C .
$$

This shows that

$$
\lim _{\varphi(w) \rightarrow \partial \mathbb{D}} \frac{\left(1-|w|^{2}\right)^{\beta}}{\left(1-|\varphi(w)|^{2}\right)^{2}}\left|\psi^{\prime}(w) \varphi^{\prime}(w)^{2}+\psi(w) \varphi^{\prime}(w) \varphi^{\prime \prime}(w)\right| \exp \frac{c}{\left(1-|\varphi(w)|^{2}\right)^{\alpha+2}}=0 .
$$

The proof of the implication is finished. 


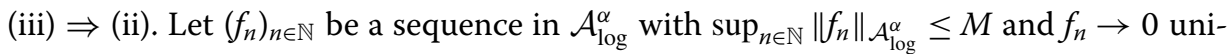
formly on every compact subset of $\mathbb{D}$ as $n \rightarrow \infty$. We have, for all $c>0$, and especially for constant $C$ in Lemma 2.2, for any $\varepsilon>0$, the result that there exists a constant $\delta \in(0,1)$ such that whenever $\delta<|\varphi(z)|<1$, it follows that

$$
\begin{aligned}
& \left(1-|z|^{2}\right)^{\beta}\left|\psi^{\prime \prime \prime}(z)\right| \exp \frac{C}{\left(1-|\varphi(z)|^{2}\right)^{\alpha+2}}<\varepsilon, \\
& \frac{\left(1-|z|^{2}\right)^{\beta}}{\left(1-|\varphi(z)|^{2}\right)^{3}}\left|\varphi^{\prime}(z)\right|^{3}|\psi(z)| \exp \frac{C}{\left(1-|\varphi(z)|^{2}\right)^{\alpha+2}}<\varepsilon, \\
& \frac{\left(1-|z|^{2}\right)^{\beta}}{\left(1-|\varphi(z)|^{2}\right)^{2}}\left|\psi^{\prime}(z) \varphi^{\prime}(z)^{2}+\psi(z) \varphi^{\prime}(z) \varphi^{\prime \prime}(z)\right| \exp \frac{C}{\left(1-|\varphi(z)|^{2}\right)^{\alpha+2}}<\varepsilon,
\end{aligned}
$$

and

$$
\frac{\left(1-|z|^{2}\right)^{\beta}}{1-|\varphi(z)|^{2}}\left|\psi(z) \varphi^{\prime \prime \prime}(z)+3 \psi^{\prime \prime}(z) \varphi^{\prime}(z)+3 \psi^{\prime}(z) \varphi^{\prime \prime}(z)\right| \exp \frac{C}{\left(1-|\varphi(z)|^{2}\right)^{\alpha+2}}<\varepsilon .
$$

Then by Lemma 2.2, we have

$$
\begin{aligned}
& \left\|\mathcal{D} W_{\varphi, \psi} f_{n}\right\|_{\mathcal{Z}_{\beta}} \\
& =\left|\left(\psi \cdot f_{n} \circ \varphi\right)^{\prime}(0)\right|+\left|\left(\psi \cdot f_{n} \circ \varphi\right)^{\prime \prime}(0)\right|+\sup _{z \in \mathbb{D}}\left(1-|z|^{2}\right)^{\beta}\left|\left(\psi \cdot f_{n} \circ \varphi\right)^{\prime \prime \prime}(z)\right| \\
& \leq\left|\left(\psi \cdot f_{n} \circ \varphi\right)^{\prime}(0)\right|+\left|\left(\psi \cdot f_{n} \circ \varphi\right)^{\prime \prime}(0)\right|+\sup _{z \in \mathbb{D}}\left(1-|z|^{2}\right)^{\beta}\left|\psi^{\prime \prime \prime}(z) f_{n}(\varphi(z))\right| \\
& +\sup _{z \in \mathbb{D}}\left(1-|z|^{2}\right)^{\beta}\left|\left(3 \psi^{\prime \prime}(z) \varphi^{\prime}(z)+3 \psi^{\prime}(z) \varphi^{\prime \prime}(z)+\psi(z) \varphi^{\prime \prime \prime}(z)\right) f_{n}^{\prime}(\varphi(z))\right| \\
& +3 \sup _{z \in \mathbb{D}}\left(1-|z|^{2}\right)^{\beta}\left|\left(\psi^{\prime}(z) \varphi^{\prime}(z)^{2}+\psi(z) \varphi^{\prime}(z) \varphi^{\prime \prime}(z)\right) f_{n}^{\prime \prime}(\varphi(z))\right| \\
& +\sup _{z \in \mathbb{D}}\left(1-|z|^{2}\right)^{\beta}\left|\psi(z) \varphi^{\prime}(z)^{3} f_{n}^{\prime \prime \prime}(\varphi(z))\right| \\
& \leq\left|\left(\psi \cdot f_{n} \circ \varphi\right)^{\prime}(0)\right|+\left|\left(\psi \cdot f_{n} \circ \varphi\right)^{\prime \prime}(0)\right|+\sup _{|\varphi(z)| \leq \delta}\left(1-|z|^{2}\right)^{\beta}\left|\psi^{\prime \prime \prime}(z) f_{n}(\varphi(z))\right| \\
& +\sup _{\delta<|\varphi(z)|<1}\left(1-|z|^{2}\right)^{\beta}\left|\psi^{\prime \prime \prime}(z) f_{n}(\varphi(z))\right| \\
& +\sup _{|\varphi(z)| \leq \delta}\left(1-|z|^{2}\right)^{\beta}\left|\left(3 \psi^{\prime \prime}(z) \varphi^{\prime}(z)+3 \psi^{\prime}(z) \varphi^{\prime \prime}(z)+\psi(z) \varphi^{\prime \prime \prime}(z)\right) f_{n}^{\prime}(\varphi(z))\right| \\
& +\sup _{\delta<|\varphi(z)|<1}\left(1-|z|^{2}\right)^{\beta}\left|\left(3 \psi^{\prime \prime}(z) \varphi^{\prime}(z)+3 \psi^{\prime}(z) \varphi^{\prime \prime}(z)+\psi(z) \varphi^{\prime \prime \prime}(z)\right) f_{n}^{\prime}(\varphi(z))\right| \\
& +3 \sup _{|\varphi(z)| \leq \delta}\left(1-|z|^{2}\right)^{\beta}\left|\left(\psi^{\prime}(z) \varphi^{\prime}(z)^{2}+\psi(z) \varphi^{\prime}(z) \varphi^{\prime \prime}(z)\right) f_{n}^{\prime \prime}(\varphi(z))\right| \\
& +3 \sup _{\delta<|\varphi(z)|<1}\left(1-|z|^{2}\right)^{\beta}\left|\left(\psi^{\prime}(z) \varphi^{\prime}(z)^{2}+\psi(z) \varphi^{\prime}(z) \varphi^{\prime \prime}(z)\right) f_{n}^{\prime \prime}(\varphi(z))\right| \\
& +\sup _{|\varphi(z)| \leq \delta}\left(1-|z|^{2}\right)^{\beta}\left|\psi(z) \varphi^{\prime}(z)^{3} f_{n}^{\prime \prime \prime}(\varphi(z))\right|+\sup _{\delta<|\varphi(z)|<1}\left(1-|z|^{2}\right)^{\beta}\left|\psi(z) \varphi^{\prime}(z)^{3} f_{n}^{\prime \prime \prime}(\varphi(z))\right| \\
& \leq\left|\left(\psi \cdot f_{n} \circ \varphi\right)^{\prime}(0)\right|+\left|\left(\psi \cdot f_{n} \circ \varphi\right)^{\prime \prime}(0)\right|+\left\|\psi^{\prime}\right\|_{\mathcal{Z}_{\beta}} \sup _{|\varphi(z)| \leq \delta}\left|f_{n}(\varphi(z))\right| \\
& +\sup _{\delta<|\varphi(z)|<1}\left(1-|z|^{2}\right)^{\beta}\left|\psi^{\prime \prime \prime}(z)\right| \exp \frac{C}{\left(1-|\varphi(z)|^{2}\right)^{\alpha+2}}+M_{0} \sup _{|\varphi(z)| \leq \delta}\left|f_{n}^{\prime}(\varphi(z))\right|
\end{aligned}
$$




$$
\begin{aligned}
& +\sup _{\delta<|\varphi(z)|<1} \frac{\left(1-|z|^{2}\right)^{\beta}}{1-|\varphi(z)|^{2}}\left|3 \psi^{\prime \prime}(z) \varphi^{\prime}(z)+3 \psi^{\prime}(z) \varphi^{\prime \prime}(z)+\psi(z) \varphi^{\prime \prime \prime}(z)\right| \exp \frac{C}{\left(1-|\varphi(z)|^{2}\right)^{\alpha+2}} \\
& +3 \sup _{\delta<|\varphi(z)|<1} \frac{\left(1-|z|^{2}\right)^{\beta}}{\left(1-|\varphi(z)|^{2}\right)^{2}}\left|\psi^{\prime}(z) \varphi^{\prime}(z)^{2}+\psi(z) \varphi^{\prime}(z) \varphi^{\prime \prime}(z)\right| \exp \frac{C}{\left(1-|\varphi(z)|^{2}\right)^{\alpha+2}} \\
& +3 M_{2} \sup _{|\varphi(z)| \leq \delta}\left|f_{n}^{\prime \prime}(\varphi(z))\right|+M_{1} \sup _{|\varphi(z)| \leq \delta}\left|f_{n}^{\prime \prime \prime}(\varphi(z))\right| \\
& +\sup _{\delta<|\varphi(z)|<1} \frac{\left(1-|z|^{2}\right)^{\beta}}{\left(1-|\varphi(z)|^{2}\right)^{3}}|\psi(z)|\left|\varphi^{\prime}(z)\right|^{3} \exp \frac{C}{\left(1-|\varphi(z)|^{2}\right)^{\alpha+2}} .
\end{aligned}
$$

By Cauchy's estimation, if $\left(f_{n}\right)_{n \in \mathbb{N}}$ converges to zero on each compact subset of $\mathbb{D}$, then $\left(f_{n}^{\prime}\right)_{n \in \mathbb{N}},\left(f_{n}^{\prime \prime}\right)_{n \in \mathbb{N}}$, and $\left(f_{n}^{\prime \prime \prime}\right)_{n \in \mathbb{N}}$ also do as $n \rightarrow \infty$. From this, and since both $\{z \in \mathbb{D}:|z| \leq \delta\}$ and $\{0\}$ are compact subset of $\mathbb{D}$, we can choose a natural number $N$ such that, whenever $n>N$, it follows that

$$
\left|\left(\psi \cdot f_{n} \circ \varphi\right)^{\prime}(0)\right|+\left|\left(\psi \cdot f_{n} \circ \varphi\right)^{\prime \prime}(0)\right|<\varepsilon
$$

and

$$
\sup _{|\varphi(z)| \leq \delta}\left|f_{n}^{(i)}(\varphi(z))\right|<\varepsilon
$$

where $i=0,1,2,3$. Consequently, for all $n>N$ it follows that

$$
\left\|\mathcal{D} W_{\varphi, \psi} f_{n}\right\|_{\mathcal{Z}_{\beta}} \leq\left(7+\left\|\psi^{\prime}\right\|_{\mathcal{Z}_{\beta}}+M_{0}+M_{1}+3 M_{2}\right) \varepsilon
$$

which shows that the operator $\mathcal{D} W_{\varphi, \psi}: \mathcal{A}_{\log }^{\alpha} \rightarrow \mathcal{Z}_{\beta}$ is compact.

(ii) $\Rightarrow$ (i). This implication is obvious. The proof is finished.

Now, we consider the boundedness of operator $\mathcal{D} W_{\varphi, \psi}: \mathcal{A}_{\log }^{\alpha} \rightarrow \mathcal{Z}_{\beta, 0}$. We first have the following result.

Lemma 2.4 Let $\alpha>-1$ and $\beta>0$. Let $\varphi$ be an analytic self-map of $\mathbb{D}$ and $\psi$ an analytic function on $\mathbb{D}$. Then, for all $c>0$, the following statements are equivalent:

(i)

$$
\begin{aligned}
& \lim _{z \rightarrow \partial \mathbb{D}} \frac{\left(1-|z|^{2}\right)^{\beta}}{1-|\varphi(z)|^{2}}\left|\psi(z) \varphi^{\prime \prime \prime}(z)+3 \psi^{\prime \prime}(z) \varphi^{\prime}(z)+3 \psi^{\prime}(z) \varphi^{\prime \prime}(z)\right| \\
& \quad \times \exp \frac{c}{\left(1-|\varphi(z)|^{2}\right)^{\alpha+2}}=0 .
\end{aligned}
$$

(ii)

$$
\begin{aligned}
& \lim _{\varphi(z) \rightarrow \partial \mathbb{D}} \frac{\left(1-|z|^{2}\right)^{\beta}}{1-|\varphi(z)|^{2}}\left|\psi(z) \varphi^{\prime \prime \prime}(z)+3 \psi^{\prime \prime}(z) \varphi^{\prime}(z)+3 \psi^{\prime}(z) \varphi^{\prime \prime}(z)\right| \\
& \quad \times \exp \frac{c}{\left(1-|\varphi(z)|^{2}\right)^{\alpha+2}}=0,
\end{aligned}
$$

and $\psi \varphi^{\prime \prime \prime}+3 \psi^{\prime \prime} \varphi^{\prime}+3 \psi^{\prime} \varphi^{\prime \prime} \in \mathcal{A}_{\beta, 0}$. 
Proof Suppose that (i) holds. Since

$$
\frac{1}{1-|\varphi(z)|^{2}} \exp \frac{c}{\left(1-|\varphi(z)|^{2}\right)^{\alpha+2}} \geq 1
$$

for all $z \in \mathbb{D}$, we have

$$
\begin{aligned}
& \left(1-|z|^{2}\right)^{\beta}\left|\psi(z) \varphi^{\prime \prime \prime}(z)+3 \psi^{\prime \prime}(z) \varphi^{\prime}(z)+3 \psi^{\prime}(z) \varphi^{\prime \prime}(z)\right| \\
& \quad \leq \frac{\left(1-|z|^{2}\right)^{\beta}}{1-|\varphi(z)|^{2}}\left|\psi(z) \varphi^{\prime \prime \prime}(z)+3 \psi^{\prime \prime}(z) \varphi^{\prime}(z)+3 \psi^{\prime}(z) \varphi^{\prime \prime}(z)\right| \exp \frac{c}{\left(1-|\varphi(z)|^{2}\right)^{\alpha+2}} \\
& \quad \rightarrow 0,
\end{aligned}
$$

as $z \rightarrow \partial \mathbb{D}$. Hence $\psi \varphi^{\prime \prime \prime}+3 \psi^{\prime \prime} \varphi^{\prime}+3 \psi^{\prime} \varphi^{\prime \prime} \in \mathcal{A}_{\beta, 0}$. Since $\varphi(z) \rightarrow \partial \mathbb{D}$ implies $z \rightarrow \partial \mathbb{D}$, it follows that the first assertion in (ii) holds.

Now suppose that (ii) holds, but (i) is not true. Then there exist constants $c_{0}>0, \varepsilon_{0}>0$ and a sequence $\left\{z_{n}\right\}$ tending to $\partial \mathbb{D}$ as $n \rightarrow \infty$ such that

$$
\frac{\left(1-\left|z_{n}\right|^{2}\right)^{\beta}}{1-\left|\varphi\left(z_{n}\right)\right|^{2}}\left|\psi(z) \varphi^{\prime \prime \prime}(z)+3 \psi^{\prime \prime}(z) \varphi^{\prime}(z)+3 \psi^{\prime}(z) \varphi^{\prime \prime}(z)\right| \exp \frac{c}{\left(1-\left|\varphi\left(z_{n}\right)\right|^{2}\right)^{\alpha+2}} \geq \varepsilon_{0} .
$$

Since $\psi \varphi^{\prime \prime \prime}+3 \psi^{\prime \prime} \varphi^{\prime}+3 \psi^{\prime} \varphi^{\prime \prime} \in \mathcal{A}_{\beta, 0}$, it follows from (20) that the sequence $\left(z_{n}\right)_{n \in \mathbb{N}}$ has a subsequence $\left(z_{n_{k}}\right)_{k \in \mathbb{N}}$ with $\varphi\left(z_{n_{k}}\right) \rightarrow \partial \mathbb{D}$. Therefore, applying $\left(z_{n_{k}}\right)_{k \in \mathbb{N}}$ to the first assertion in (ii), we arrive at a contradiction to (20), finishing the proof.

By Lemma 2.4, the following result follows similar to the proof of Theorem 2.3. Hence, we omit the details.

Theorem 2.5 Let $\alpha>-1$ and $\beta>0$. Let $\varphi$ be an analytic self-map of $\mathbb{D}$ and $\psi$ an analytic function on $\mathbb{D}$. Then, for all $c>0$, the following statements are equivalent:

(i) The operator $\mathcal{D} W_{\varphi, \psi}: \mathcal{A}_{\log }^{\alpha} \rightarrow \mathcal{Z}_{\beta, 0}$ is bounded.

(ii) The operator $\mathcal{D} W_{\varphi, \psi}: \mathcal{A}_{\log }^{\alpha} \rightarrow \mathcal{Z}_{\beta, 0}$ is compact.

(iii)

$$
\begin{aligned}
& \psi \varphi^{\prime \prime \prime}+3 \psi^{\prime \prime} \varphi^{\prime}+3 \psi^{\prime} \varphi^{\prime \prime}, \varphi^{\prime 3} \psi, \psi^{\prime} \varphi^{\prime 2}+\psi \varphi^{\prime} \varphi^{\prime \prime}, \psi^{\prime \prime \prime} \in \mathcal{A}_{\beta, 0}, \\
& \lim _{\varphi(z) \rightarrow \partial \mathbb{D}}\left(1-|z|^{2}\right)^{\beta}\left|\psi^{\prime \prime \prime}(z)\right| \exp \frac{c}{\left(1-|\varphi(z)|^{2}\right)^{\alpha+2}}=0, \\
& \lim _{\varphi(z) \rightarrow \partial \mathbb{D}} \frac{\left(1-|z|^{2}\right)^{\beta}}{1-|\varphi(z)|^{2}}\left|\psi(z) \varphi^{\prime \prime \prime}(z)+3 \psi^{\prime \prime}(z) \varphi^{\prime}(z)+3 \psi^{\prime}(z) \varphi^{\prime \prime}(z)\right| \\
& \quad \times \exp \frac{c}{\left(1-|\varphi(z)|^{2}\right)^{\alpha+2}}=0, \\
& \lim _{\varphi(z) \rightarrow \partial \mathbb{D}} \frac{\left(1-|z|^{2}\right)^{\beta}}{\left(1-|\varphi(z)|^{2}\right)^{2}}\left|\psi^{\prime}(z) \varphi^{\prime}(z)^{2}+\psi(z) \varphi^{\prime}(z) \varphi^{\prime \prime}(z)\right| \exp \frac{c}{\left(1-|\varphi(z)|^{2}\right)^{\alpha+2}}=0,
\end{aligned}
$$

and

$$
\lim _{\varphi(z) \rightarrow \partial \mathbb{D}} \frac{\left(1-|z|^{2}\right)^{\beta}}{\left(1-|\varphi(z)|^{2}\right)^{3}}|\psi(z)|\left|\varphi^{\prime}(z)\right|^{3} \exp \frac{c}{\left(1-|\varphi(z)|^{2}\right)^{\alpha+2}}=0 .
$$


(iv)

$$
\begin{aligned}
& \lim _{z \rightarrow \partial \mathbb{D}}\left(1-|z|^{2}\right)^{\beta}\left|\psi^{\prime \prime \prime}(z)\right| \exp \frac{c}{\left(1-|\varphi(z)|^{2}\right)^{\alpha+2}}=0, \\
& \lim _{z \rightarrow \partial \mathbb{D}} \frac{\left(1-|z|^{2}\right)^{\beta}}{1-|\varphi(z)|^{2}}\left|\psi(z) \varphi^{\prime \prime \prime}(z)+3 \psi^{\prime \prime}(z) \varphi^{\prime}(z)+3 \psi^{\prime}(z) \varphi^{\prime \prime}(z)\right| \\
& \quad \times \exp \frac{c}{\left(1-|\varphi(z)|^{2}\right)^{\alpha+2}}=0, \\
& \lim _{z \rightarrow \partial \mathbb{D}} \frac{\left(1-|z|^{2}\right)^{\beta}}{\left(1-|\varphi(z)|^{2}\right)^{2}}\left|\psi^{\prime}(z) \varphi^{\prime}(z)^{2}+\psi(z) \varphi^{\prime}(z) \varphi^{\prime \prime}(z)\right| \exp \frac{c}{\left(1-|\varphi(z)|^{2}\right)^{\alpha+2}}=0,
\end{aligned}
$$

and

$$
\lim _{z \rightarrow \partial \mathbb{D}} \frac{\left(1-|z|^{2}\right)^{\beta}}{\left(1-|\varphi(z)|^{2}\right)^{3}}|\psi(z)|\left|\varphi^{\prime}(z)\right|^{3} \exp \frac{c}{\left(1-|\varphi(z)|^{2}\right)^{\alpha+2}}=0
$$

\section{Competing interests}

The author declares that they have no competing interests.

\section{Acknowledgements}

The author would like to thank the referee for his or her helpful comments and suggestions. This work is supported by the National Natural Science Foundation of China (Grant No. 11201323), the Sichuan Province University Key Laboratory of Bridge Non-destruction Detecting and Engineering Computing (Grant No. 2013QZJ01, No. 2013QYY01), the Key Fund Project of Sichuan Provincial Department of Education (Grant No. 12ZB288) and the Introduction of Talent Project of SUSE (Grant No. 2014RC04).

Received: 7 March 2014 Accepted: 25 September 2014 Published: 16 Oct 2014

\section{References}

1. Jiang, ZJ, Cao, GF: Composition operator on Bergman-Orlicz space. J. Inequal. Appl. 2009, Article ID 832686 (2009)

2. Jiang, ZJ: Carleson measures and composition operators on Bergman-Orlicz spaces of the unit ball. Int. J. Math. Anal. 4(33), 1607-1615 (2010)

3. Stević, S: Weighted composition operators from Bergman-Privalov-type spaces to weighted-type spaces on the unit ball. Appl. Math. Comput. 217, 1939-1943 (2010)

4. Yang, W, Yan, W: Generalized weighted composition operators from area Nevanlinna spaces to weighted-type spaces. Bull. Korean Math. Soc. 48(6), 1195-1205 (2011)

5. Zhu, K: Spaces of Holomorphic Functions in the Unit Ball. Springer, New York (2005)

6. Jiang, ZJ: Weighted composition operator from Bergman-type spaces into Bers-type spaces. Acta Math. Sci. 53(1), 67-74 (2010) (in Chinese)

7. Jiang, ZJ: Weighted composition operators from weighted Bergman spaces to some spaces of analytic functions on the upper half plane. Ars Comb. 91, 10-16 (2010)

8. Jiang, ZJ: On Volterra composition operators from Bergman-type space to Bloch-type space. Czechoslov. Math. J. 61(4), 993-1005 (2011)

9. Jiang, ZJ, Stević, S: Compact differences of weighted composition operators from weighted Bergman spaces to weighted-type spaces. Appl. Math. Comput. 217, 3522-3530 (2010)

10. Li, S, Stević, S: Composition followed by differentiation between Bloch type spaces. J. Comput. Anal. Appl. 9(2), 195-205 (2007)

11. Li, S, Stević, S: Weighted composition operators from Bergman-type spaces into Bloch spaces. Proc. Indian Acad. Sci. Math. Sci. 117(3), 371-385 (2007)

12. Li, S, Stević, S: Weighted composition operators from $\alpha$-Bloch space to $H^{\infty}$ on the polydisk. Numer. Funct. Anal. Optim. 28(7), 911-925 (2007)

13. Li, S, Stević, S: Weighted composition operators from $H^{\infty}$ to the Bloch space on the polydisc. Abstr. Appl. Anal. 2007, Article ID 48478 (2007)

14. Li, S, Stević, S: Weighted composition operators between $H^{\infty}$ and $\alpha$-Bloch spaces in the unit ball. Taiwan. J. Math. 12, 1625-1639 (2008)

15. Li, S, Stević, S: Composition followed by differentiation from mixed norm spaces to $\alpha$-Bloch spaces. Sb. Math. 199(12), 1847-1857 (2008)

16. Li, S, Stević, S: Generalized composition operators on Zygmund spaces and Bloch type spaces. J. Math. Anal. Appl. $338,1282-1295(2008)$

17. Li, S, Stević, S: Weighted composition operators from Zygmund spaces into Bloch spaces. Appl. Math. Comput. 206(2), 825-831 (2008)

18. Li, S, Stević, S: Products of Volterra type operator and composition operator from $H^{\infty}$ and Bloch spaces to the Zygmund space. J. Math. Anal. Appl. 345, 40-52 (2008) 
19. Li, S, Stević, S: Composition followed by differentiation between $H^{\infty}$ and $\alpha$-Bloch spaces. Houst. J. Math. 35(1), 327-340 (2009)

20. Li, S, Stević, S: Products of composition and differentiation operators from Zygmund spaces to Bloch spaces and Bers spaces. Appl. Math. Comput. 217, 3144-3154 (2010)

21. Nieminen, PJ: Compact differences of composition operators on Bloch and Lipschitz spaces. Comput. Methods Funct. Theory 7(2), 325-344 (2007)

22. Ohno, S: Weighted composition operators between $H^{\infty}$ and the Bloch space. Taiwan. J. Math. 5(3), 555-563 (2001)

23. Sharma, SD, Sharma, AK, Ahmed, S: Composition operators between Hardy and Bloch-type spaces of the upper half-plane. Bull. Korean Math. Soc. 43(3), 475-482 (2007)

24. Stević, S: Composition operators between $H^{\infty}$ and the $\alpha$-Bloch spaces on the polydisc. Z. Anal. Anwend. 25, 457-466 (2006)

25. Stević, S: Essential norms of weighted composition operators from the $\alpha$-Bloch space to a weighted-type space on the unit ball. Abstr. Appl. Anal. 2008, Article ID 279691 (2008)

26. Stević, S: Norm of weighted composition operators from Bloch space to $H^{\infty}$ on the unit ball. Ars Comb. 88, 125-127 (2008)

27. Stević, S: Norms of some operators from Bergman spaces to weighted and Bloch-type space. Util. Math. 76, 59-64 (2008)

28. Stević, S: On a new operator from $H^{\infty}$ to the Bloch-type space on the unit ball. Util. Math. 77, 257-263 (2008)

29. Stević, S: On a new operator from the logarithmic Bloch space to the Bloch-type space on the unit ball. Appl. Math. Comput. 206, 313-320 (2008)

30. Stević, S: Integral-type operators from the mixed-norm space to the Bloch-type space on the unit ball. Sib. Math. J. 50(6), 1098-1105 (2009)

31. Stević, S: Norm and essential norm of composition followed by differentiation from $\alpha$-Bloch spaces to $H^{\infty}$. Appl. Math. Comput. 207, 225-229 (2009)

32. Stević, S: Norm of weighted composition operators from $\alpha$-Bloch spaces to weighted-type spaces. Appl. Math Comput. 215, 818-820 (2009)

33. Stević, S: On a new integral-type operator from the Bloch spaces to Bloch-type spaces on the unit ball. J. Math. Anal. Appl. 354, 426-434 (2009)

34. Stević, S: Weighted composition operators from weighted Bergman spaces to weighted-type spaces on the unit ball. Appl. Math. Comput. 212, 499-504 (2009)

35. Stević, S: Weighted differentiation composition operators from $H^{\infty}$ and Bloch spaces to $n$-th weighted-type spaces on the unit disk. Appl. Math. Comput. 216, 3634-3641 (2010)

36. Stević, S, Jiang, ZJ: Differences of weighted composition operators on the unit polydisk. Sib. Math. J. 52(2), 454-468 (2011)

37. Stević, S, Agarwal, RP: Weighted composition operators from logarithmic Bloch-type spaces to Bloch-type spaces. J. Inequal. Appl. 2009, Article ID 964814 (2009)

38. Yang, W: Weighted composition operators from Bloch-type spaces to weighted-type spaces. Ars Comb. 93, 265-274 (2009)

39. Sharma, AK, Abbas, Z: Weighted composition operators between weighted Bergman-Nevanlinna and Bloch-type spaces. Appl. Math. Sci. 41(4), 2039-2048 (2010)

40. Kumar, P, Sharma, SD: Weighted composition operators from weighted Bergman Nevanlinna spaces to Zygmund spaces. Int. J. Mod. Math. Sci. 3(1), 31-54 (2012)

41. Hibschweiler, RA, Portnoy, N: Composition followed by differentiation between Bergman and Hardy spaces. Rocky Mt. J. Math. 35(3), 843-855 (2005)

42. Hosokawa, T, Ohno, S: Differences of composition operators on the Bloch spaces. J. Oper. Theory 57, 229-242 (2007)

43. Ohno, S: Products of composition and differentiation on Bloch spaces. Bull. Korean Math. Soc. 46(6), 1135-1140 (2009)

44. Stević, S: Products of composition and differentiation operators on the weighted Bergman space. Bull. Belg. Math. Soc. Simon Stevin 16, 623-635 (2009)

45. Stević, S: Composition followed by differentiation from $H^{\infty}$ and the Bloch space to $n$-th weighted-type spaces on the unit disk. Appl. Math. Comput. 216, 3450-3458 (2010)

46. Sharma, AK: Products of composition multiplication and differentiation between Bergman and Bloch type spaces Turk. J. Math. 35, 275-291 (2011)

47. Stević, S, Sharma, AK, Bhat, A: Products of multiplication composition and differentiation operators on weighted Bergman spaces. Appl. Math. Comput. 217, 8115-8125 (2011)

48. Stević, S, Sharma, AK, Bhat, A: Essential norm of multiplication composition and differentiation operators on weighted Bergman spaces. Appl. Math. Comput. 218, 2386-2397 (2011)

49. Jiang, ZJ: On a class of operators from weighted Bergman spaces to some spaces of analytic functions. Taiwan. J. Math. 15(5), 2095-2121 (2011)

50. Cowen, CC, MacCluer, BD: Composition Operators on Spaces of Analytic Functions. CRC Press, Boca Raton (1995)

10.1186/1029-242X-2014-404

Cite this article as: Jiang: On a product operator from weighted Bergman-Nevanlinna spaces to weighted Zygmund spaces. Journal of Inequalities and Applications 2014, 2014:404 